\title{
Rugosidade da superfície de madeiras amazônicas
}

\author{
Surface roughness of Amazonian woods
}

\author{
Keiti Mendes Pereira ${ }^{1}$, Rosilei Aparecida Garcia ${ }^{2}$ e Alexandre Miguel do Nascimento ${ }^{3}$
}

\begin{abstract}
Resumo
A rugosidade é um parâmetro importante na avaliação da qualidade da superfície da madeira pois afeta o desempenho de adesivos e revestimentos e, consequentemente, a qualidade do produto final. A rugosidade depende das operações de usinagem, da estrutura anatômica da madeira e da combinação entre ambas. O objetivo deste estudo foi avaliar o efeito das condições de preparo da superfície (aplainamento e lixamento) e da estrutura anatômica na rugosidade superficial de oito espécies de madeiras amazônicas. As madeiras estudadas foram: abiurana (Pouteria guianensis), garapeira (Apuleia molaris), jequitibá (Cariniana sp.), cedro (Cedrela odorata), angelim (Parkia pendula), angelim pedra (Hymenolobium excelsum), cerejeira (Amburana acreana) e cumaru (Dipteryx odorata). As madeiras foram classificadas de acordo com a textura (fina, média e grossa) e a densidade aparente determinada segundo a ASTM D 2395-93. Análises anatômicas foram realizadas pela aquisição de imagens digitais e os vasos mensurados pelo software Image-Pro ${ }^{\circledR}$ Plus. A rugosidade superficial foi determinada pelo rugosímetro de agulha modelo TR 200 segundo a norma NBR ISO 4287:2002 em que foram obtidos os parâmetros de amplitude $\left(R_{\mathrm{a}}, R_{\mathrm{z}}, \mathrm{R}_{\mathrm{t}}\right.$, $R_{p}$ e $R_{v}$ ). A textura da madeira afetou significativamente a rugosidade superficial, principalmente na superfície lixada. O lixamento diminuiu a rugosidade superficial das madeiras para todos os parâmetros, exceto para o parâmetro $R_{v}$ das madeiras de angelim pedra e cumaru.
\end{abstract}

Palavras-chave: parâmetros de amplitude, textura da madeira, usinagem.

\begin{abstract}
Roughness is an important parameter to evaluate the quality of wood surface because it affects the adhesive and coating performance and thus the quality of the final product. The roughness depends on the machining operations, wood anatomical features and combination of both. The objective of this study was to evaluate the effect of surface preparation conditions (planing and sanding) and the anatomical structure on the surface roughness of the eight Amazonian wood species. The studied woods were: abiurana (Pouteria guianensis), garapeira (Apuleia molaris), jequitibá (Cariniana sp.), cedro (Cedrela odorata), angelim (Parkia pendula), angelim pedra (Hymenolobium excelsum), cerejeira (Amburana acreana) and cumaru (Dipteryx odorata). Woods were classified according to the texture (fine, medium and coarse) and the apparent density determined following the ASTM D 2395-93 norms. Anatomical analyses were performed by acquisition of digital images and vessel parameters measured by the Image-Pro ${ }^{\circledR}$ Plus software. Surface roughness was determined by the needle type roughmeter model TR 200 according to NBR ISO 4287:2002, and amplitude parameters $\left(R_{a}, R_{z}, R_{t}, R_{p}\right.$ and $\left.R_{v}\right)$ were obtained. Wood texture affected significantly surface roughness, principally in sanded surfaces. Sanding reduced the surface roughness for all parameters, except for the $R_{v}$ parameter of angelim pedra and cumaru woods.
\end{abstract}

Keywords: amplitude parameters, wood texture, machining.

\section{INTRODUÇÃO}

A rugosidade caracteriza as irregularidades da superfície da madeira e afeta diretamente a aplicação e a durabilidade dos revestimentos. Estas irregularidades podem ser determinadas pelas medições da altura, largura e forma dos picos e vales ocasionados durante o processo de usinagem ou pela própria estrutura anatômica da madeira (MAGOSS, 2008). A rugosidade superficial é um crité-

\footnotetext{
${ }_{1}$ Professora Mestra do Departamento de Ciências Agrárias. UFC - Universidade Federal do Acre. Estrada Dias Martins, BR 364 - Km 04 - 69915-900 - Rio Branco, AC, Brasil. E-mail: keiti.roseani@gmail.com.

2Professora Associada do Departamento de Produtos Florestais. UFRRJ - Universidade Federal Rural do Rio de Janeiro, Instituto de Florestas. BR 465 - Km 07 - Campus de Seropédica - 23890000 - Seropédica, RJ, Brasil. E-mail: rosileigar@hotmail.com.

3Professor Titular do Departamento de Produtos Florestais. UFRRJ - Universidade Federal Rural do Rio de Janeiro, Instituto de Florestas. BR 465 - Km 07 - Campus de Seropédica - 23890000 - Seropédica, RJ, Brasil. E-mail: alexmnasci@gmail.com.
}

Sci. For., Piracicaba, v. 46, n. 119, p. 347-356, set. 2018 DOI: dx.doi.org/10.18671/scifor.v46n119.02 
rio importante para avaliar as condições das ferramentas, o desempenho da usinagem e a qualidade do produto. Vários trabalhos procuraram avaliar o efeito da usinagem na rugosidade superficial (KILIC et al., 2006; MARTINS et al., 2011). Kilic et al. (2006), estudando o efeito da usinagem na rugosidade superficial das madeiras de Fagus orientalis e Populus tremula, observaram uma diminuição da rugosidade com o aumento do número de grão da lixa. Entretanto, no trabalho realizado por Martins et al. (2011), as operações de usinagem (aplainamento x lixamento) não afetaram significativamente a rugosidade da superfície da madeira de Eucalyptus benthamii. Esses diferentes resultados podem ser explicados pela estrutura celular particular de cada espécie de madeira demonstrando a importância de se estudar o efeito da usinagem associada à estrutura anatômica. Segundo Csanády et al. (2015), a maior parte da rugosidade resulta da estrutura anatômica da madeira. A presença de grãs irregulares, sílica, nós, a porosidade e dimensões de fibras e vasos interferem diretamente nas características da superfície (BAJIC et al., 2008). A variação do tamanho e da quantidade de poros entre espécies ou mesmo dentro de mesma espécie de madeira afetam a rugosidade da superfície podendo ocasionar grandes vales devido à sua remoção durante o processo de usinagem. A posição do plano de corte dos vasos também afeta a rugosidade, pois as cavidades são cortadas em diferentes ângulos e posições em relação à superfície, produzindo vales de diferentes formas e tamanhos (CSANÁDY et al., 2015).

A rugosidade superficial pode ser mensurada através de vários equipamentos, os quais podem ser divididos em duas categorias: (i) por contato (ex.: apalpamento mecânico do tipo "stylus") e (ii) sem contato (ex.: interferometria de luz branca) (BHUSHAN, 2001). Os instrumentos de medição por contato são aqueles que exploram a superfície do material pelo contato direto utilizando apalpador e fornecem os desvios na forma de perfil. Esses equipamentos têm a capacidade de calcular parâmetros e emitir registros do perfil graficamente (MACHADO et al., 2009). Segundo a NBR ISO 4287 (ABNT, 2002), a superfície pode ser avaliada de forma quantitativa pelos parâmetros de rugosidade (R) e ondulação $(\mathrm{W})$. As ondulações consistem de irregularidades superficiais ou erros geométricos cujos espaçamentos são maiores que as irregularidades consideradas rugosidades (MACHADO et al., 2009). O perfil da superfície pode ser avaliado pelos parâmetros de textura superficial, que compreende os parâmetros de amplitude e de espaçamento e pelos parâmetros híbridos (PETROPOULOS, 2007). Os parâmetros de amplitude referem-se às medições dos deslocamentos verticais dos perfis; os parâmetros de espaçamento às medições de espaçamentos irregulares ao longo da superfície, independente da amplitude dessas irregularidades; e os parâmetros híbridos estão relacionados tanto com a amplitude como com o espaçamento das irregularidades superficiais (FORM TALYSURF SERIES OPERATOR'S HANDBOOK, 1985). Os parâmetros de amplitude são: rugosidade aritmética ou média $\left(R_{a}\right)$, desvio médio quadrático do perfil $\left(R_{q}\right)$, amplitude máxima de perfil por comprimento de medição $\left(R_{z}\right)$, amplitude total de perfil $\left(R_{t}\right)$, amplitude máxima de picos dentro do comprimento de ondas $\left(R_{p}\right)$, amplitude máxima de vales dentro do comprimento de ondas $\left(R_{v}\right)$ e média da altura máxima de picos em cada comprimento de ondas ( $\mathrm{R}_{\mathrm{pm}}$ ) (ISO 4287: 1997). O parâmetro $\mathrm{R}_{\mathrm{a}}$ é o mais utilizado devido ao seu baixo coeficiente de variação, sendo fornecido em equipamentos mais simples para medição da superfície de produtos de madeira. Porém, esse parâmetro não fornece valores reais de alturas de picos e vales e é extremamente sensível a presença de vasos, não sendo adequado para superfícies que apresentam uma frequência muito alta de vales ou picos. Os parâmetros de espaçamento são determinados pela largura média dos elementos do perfil $\left(\mathrm{R}_{\mathrm{Sm}}\right)$ e os parâmetros híbridos compreendem a inclinação média do perfil $\left(\mathrm{R}_{\mathrm{a}}\right)$, o desvio médio quadrático da inclinação do perfil $\left(\mathrm{R}_{\mathrm{q}}\right)$, o raio de aspereza (rp) e o comprimento desenvolvido do perfil $\left(\mathrm{R}_{\mathrm{lo}}\right)$ (PETROPOULOS, 2007). As medições sem contato por interferometria de luz branca permitem obter um perfil topográfico da superfície em 3D e têm sido bastante utilizadas recentemente para avaliar a rugosidade da madeira e compósitos à base de madeira (HERNÁNDEZ et al., 2014; UGULINO; HERNÁNDEZ, 2016; ZHONG et al., 2013).

Neste contexto, o objetivo deste estudo foi avaliar o efeito das condições de preparo da superfície (aplainamento e lixamento) e da estrutura anatômica na rugosidade superficial de oito espécies de madeiras amazônicas em que foram abordadas, principalmente, a textura e as dimensões dos vasos. 


\section{MATERIAL E MÉTODOS}

\section{Material}

Oito espécies de madeiras amazônicas (Tabela 1) foram coletadas na Floresta Macauã (floresta nativa manejada) situada no município de Sena Madureira, Acre. Foram obtidas amostras de $30 \mathrm{x}$ $75 \times 150 \mathrm{~mm}^{3}$ (espessura x largura x comprimento), as quais foram aplainadas, lixadas em uma das faces e em seguida redimensionadas em amostras de $10 \times 30 \times 150 \mathrm{~mm}^{3}$ (espessura x largura x comprimento). $\mathrm{O}$ material foi acondicionado em câmara climática à $20^{\circ} \mathrm{C}$ e $65 \%$ de umidade relativa (UR) até atingir massa constante.

Tabela 1. Lista das madeiras amazônicas em função das classes de textura.

Table 1. List of the Amazonian woods as related to texture class and grain type.

\begin{tabular}{llll}
\hline Textura & Nome vulgar & Nome científico & Família \\
\hline \multirow{3}{*}{ Fina } & Abiurana & Pouteria guianensis Aubl. & Sapotaceae \\
& Garapeira & Apuleia molaris Spruce ex Benth. & Fabaceae \\
& Jequitibá & Cariniana sp. & Lecythidaceae \\
& Cedro & Cedrela odorata L. & Meliaceae \\
& Angelim & Parkia pendula (Wild.) Benth. Ex Walp. & Fabaceae \\
& Angelim pedra & Hymenolobium excelsum Ducke & Fabaceae \\
& Cerejeira & Amburana acreana (Ducke) A. C. Sm. & Fabaceae \\
& Cumaru & Dipteryx odorata (Aubl.) Willd. & Fabaceae \\
\hline
\end{tabular}

As madeiras foram classificadas de acordo com a textura (fina, média e grossa), a qual foi caracterizada macroscopicamente quanto ao tamanho relativo das células (Tabela 1). Na classe de textura fina foram incluídas as madeiras que apresentaram uma estrutura uniforme com células geralmente pequenas enquanto que na classe de textura grossa foram incluídas as madeiras que apresentam uma estrutura composta por poros de grandes diâmetros e/ou parênquima abundante (FOREST PRODUCTS LABORATORY, 2010; CORADIN; MUÑIZ, 1992). A textura média foi considerada como uma classe intermediária.

A densidade aparente das madeiras foi determinada segundo a ASTM D 2395-93 (ASTM, 1999).

\section{Rugosidade superficial}

Os ensaios de rugosidade superficial foram realizados com o rugosímetro de agulha modelo TR 200 de acordo com a NBR ISO 4287:2002 (ABNT, 2002). As medições de rugosidade foram realizadas em cinco pontos de cada amostra no sentido perpendicular à grã nas superfícies aplainada $e$ lixada. Para cada ponto foram determinados os seguintes parâmetros de amplitude: rugosidade aritmética ou média $\left(R_{a}\right)$ : determinada em função da linha média do perfil de rugosidade; rugosidade total $\left(\mathrm{R}_{\mathrm{z}}\right)$ : correspondente à distância vertical entre o pico mais alto $\left(\mathrm{R}_{\mathrm{p}}\right)$ e o vale mais profundo $\left(\mathrm{R}_{\mathrm{v}}\right)$ no comprimento de medição, independente dos valores de rugosidade parcial; e rugosidade máxima $\left(R_{y}\right.$ ou $\left.R_{t}\right)$ : definida como o maior valor das rugosidades parciais que se apresenta no percurso de medição (cut-off). O comprimento de medição foi de $5 \mathrm{~mm}$ com comprimento de amostragem (cut-off) de 2,5 mm para todas as amostras e utilizou-se filtro Gauss.

\section{Análises estatísticas}

A análise de componentes principais (Principal Component Analysis - PCA) foi realizada pelo software Minitab ${ }^{\circledR} 16$, com o intuito de agrupar as espécies de madeira em função das características anatômicas. As variáveis anatômicas consideradas na análise de PCA foram: diâmetro tangencial mínimo dos vasos (Dtm), diâmetro tangencial médio dos vasos (DtM), diâmetro tangencial máximo dos vasos (DtMx), frequência mínima dos vasos (Fpm), frequência média dos vasos (FpM), frequência máxima dos vasos (FpMx), área dos vasos (Av) e fração vasos (Fv). Foram consideradas as médias padronizadas com desvio padrão igual a 1, para cada característica anatômica estudada. Para a análise das componentes principais utilizou-se a matriz de correlação dos dados. As combinações lineares estabelecidas foram interpretadas por meio dos autovetores normalizados e das correlações entre as variáveis originais e as componentes principais.

As análises estatísticas dos parâmetros de rugosidade foram realizadas pelo programa STATISTICA 7.0. A normalidade dos dados e a homogeneidade das variâncias foram verificadas pelos testes

Sci. For., Piracicaba, v. 46, n. 119, p. 347-356, set. 2018 DOI: dx.doi.org/10.18671/scifor.v46n119.02 
de Kolmogorov-Smirnov e Levene, respectivamente, a 95\% de probabilidade. Os dados dos parâmetros de rugosidade não atenderam os pressupostos da análise de variância, portanto, utilizou-se os testes não paramétricos de Wilcoxon-Mann-Whitney (comparação entre dois grupos independentes) e Kruskal-Wallis (comparação entre múltiplos grupos independentes).

A rugosidade superficial foi avaliada pelos seguintes parâmetros (variáveis dependentes): $R_{a^{\prime}} R_{z^{\prime}}$ $\mathrm{R}_{\mathrm{t}^{\prime}} \mathrm{R}_{\mathrm{p}^{\prime}} \mathrm{R}_{\mathrm{v}^{\prime}}$ visando comparar o preparo da superfície (aplainada e lixada) e as classes de textura (fina, média e grossa) das madeiras.

\section{RESULTADOS E DISCUSSÃO}

\section{Análises anatômicas}

A Tabela 2 apresenta a análise microscópica quantitativa dos poros das madeiras amazônicas. Os resultados do diâmetro tangencial do lume dos vasos permitiram classificá-los em vasos médios para as madeiras de abiurana, garapeira, angelim e cerejeira; e vasos grandes para as madeiras de jequitibá, cedro, angelim pedra e cumaru. Os vasos da madeira de angelim pedra apresentaram o maior diâmetro tangencial médio enquanto que os vasos da madeira de angelim apresentaram o menor diâmetro tangencial médio (Tabela 2). Quanto à frequência dos vasos, estes foram classificados como muito numerosos nas madeiras de garapeira e abiurana; numerosos nas madeiras jequitibá, cedro, angelim e cumaru; e pouco numerosos nas madeiras de angelim pedra e cerejeira.

Tabela 2. Análise microscópica quantitativa dos vasos das madeiras amazônicas.

Table 2. Quantitative microscopic analysis of Amazonian wood vessels.

\begin{tabular}{|c|c|c|c|c|c|c|c|c|}
\hline \multirow{2}{*}{ Espécie } & \multicolumn{3}{|c|}{ Diâmetro tangencial dos vasos $(\mu \mathrm{m})$} & \multicolumn{3}{|c|}{ Frequência dos vasos (vasos $/ \mathrm{mm}^{2}$ ) } & \multirow{2}{*}{$\begin{array}{c}\text { Área vasos } \\
\left(\mathrm{mm}^{2}\right)\end{array}$} & \multirow{2}{*}{$\begin{array}{c}\text { Fração } \\
\text { vasos }\end{array}$} \\
\hline & Mínimo & Média & Máximo & Mínimo & Média & Máximo & & \\
\hline Abiurana & 93,7 & 170,1 & 234,3 & 30 & 33,2 & 36 & 0,023 & 0,754 \\
\hline Garapeira & 78,1 & 171,2 & 166,6 & 24 & 36,1 & 45 & 0,023 & 0,831 \\
\hline Jequitibá & 135,4 & 205,1 & 271,1 & 12 & 16,4 & 22 & 0,033 & 0,542 \\
\hline Cedro & 136,9 & 230,1 & 333,3 & 9 & 14,0 & 22 & 0,042 & 0,582 \\
\hline Angelim & 93,8 & 148,3 & 223,8 & 13 & 19,2 & 25 & 0,017 & 0,332 \\
\hline Angelim pedra & 150,9 & 247,8 & 331,1 & 3 & 6,1 & 10 & 0,048 & 0,294 \\
\hline Cerejeira & 109,4 & 171,9 & 260,3 & 5 & 6,6 & 9 & 0,023 & 0,153 \\
\hline Cumaru & 135,3 & 219,1 & 276,1 & 10 & 16,4 & 23 & 0,038 & 0,618 \\
\hline
\end{tabular}

A Tabela 3 apresenta os valores, em módulo, das componentes principais I e II. A quantidade de informação total das variáveis retidas pelas duas componentes principais foi de $99,18 \%$ em que $76,56 \%$ correspondem à componente principal I e 22,62\% à componente principal II. Os maiores vetores observados na componente principal I são para as variáveis de frequência média dos vasos $(\mathrm{FpM}=-0,386)$, diâmetro tangencial mínimo dos vasos $(\mathrm{Dtm}=0,381)$ e diâmetro tangencial máximo dos vasos (DtMx $=0,380)$, indicando que estas são as mais importantes para agrupar as espécies de madeiras. Observando o diagrama de ordenação (Figura 1), no lado negativo da componente principal I, a variável FpM separa as madeiras de abiurana e garapeira das demais, as quais apresentam os maiores valores de frequência média de vasos (Tabela 2). No lado positivo da componente principal I, as variáveis Dtm e DtMx agrupam as madeiras de cedro, angelim pedra, cumaru e jequitibá as quais apresentam vasos de maiores diâmetros (Tabela 2). Na componente principal II, o diâmetro tangencial médio dos vasos $(\mathrm{DtM}=0,45)$ e área dos vasos $(\mathrm{Av}=0,43)$ foram as variáveis que mais se aproximaram das madeiras de cedro, angelim pedra, cumaru e jequitibá, indicando que as mesmas apresentam características para agrupar as espécies. De forma isolada, nenhuma das variáveis permitiu o agrupamento das madeiras de angelim e cerejeira às demais madeiras como pode ser observado na ordenação (Figura 1).

É possível estabelecer que as madeiras amazônicas estudadas estão divididas em três grupos: grupo I formado pelas madeiras jequitibá, cedro, angelim pedra e cumaru (madeiras com os maiores valores de diâmetro tangencial médio dos vasos); grupo II formado pelas madeiras de garapeira e abiurana (maiores valores de frequência média dos vasos) e grupo III formado pelas madeiras de angelim e cerejeira (que não apresentam características de diâmetro tangencial de vasos e frequência dos vasos que relacione com as demais madeiras). 
Tabela 3. Autovetores das componentes principais I e II obtidas pela análise da componente principal (PCA).

Table 3. Eigenvectors of the principal components I and II obtained by Principal Component Analysis (PCA).

\begin{tabular}{lcc}
\hline Variável & Componente I & Componente II \\
\hline Diâmetro tangencial mínimo dos vasos (Dtm) & 0,381 \\
Diâmetro tangencial médio dos vasos (DtM) & 0,325 & 0,448 \\
Diâmetro tangencial máximo dos vasos (DtMx) & 0,380 & 0,172 \\
Frequência mínima dos vasos (Fpm) & $-0,375$ & 0,224 \\
Frequência média dos vasos (FpM) & $-0,386$ & 0,258 \\
Frequência máxima dos vasos (FpMx) & $-0,371$ & 0,305 \\
Área vasos mm² (Av) & 0,330 & 0,432 \\
Fração vasos (Fv) & $-0,263$ & 0,562 \\
Variância explicada (\%) & 22,62 \\
Variância acumulada (\%) & 76,56 & 99,18 \\
Autovalores & 76,56 & 1,8 \\
\hline
\end{tabular}

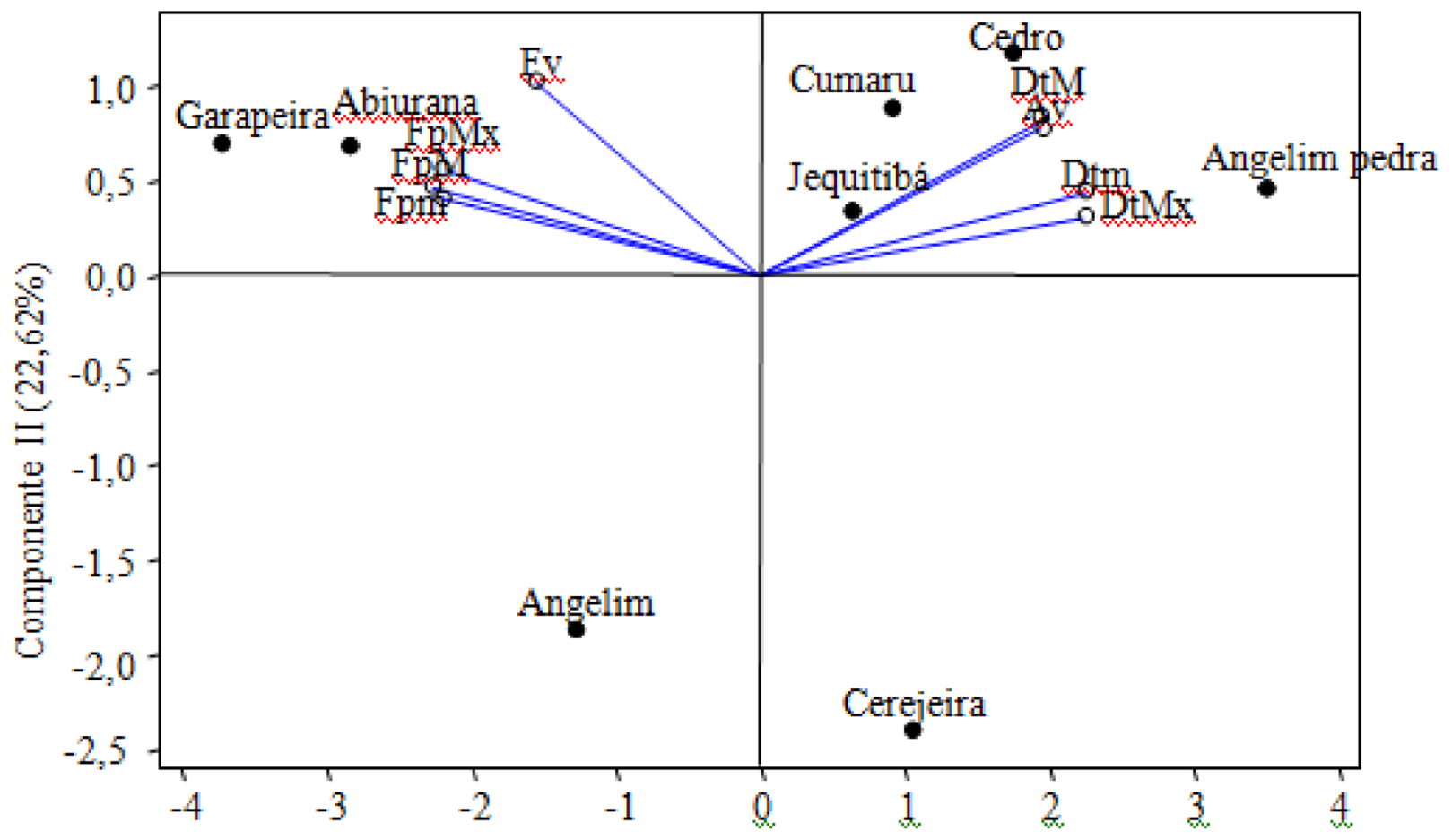

Componente I $(76,56 \%)$

Figura 1. Diagrama de ordenação da análise da componente principal (PCA). Variáveis: Dtm = diâmetro tangencial mínimo dos poros, DtM = diâmetro tangencial médio dos poros, DtMx = diâmetro tangencial máximo dos poros, $\mathrm{Fpm}=$ frequência mínima dos poros, $\mathrm{FpM}=$ frequência média dos poros, $\mathrm{FpMx}=$ frequência máxima dos poros, $A v=$ área dos vasos e $\mathrm{Fv}=$ fração vasos.

Figure 1. Principal component analysis (PCA) ordination diagram. Variables: $\mathrm{Dtm}=$ minimum tangential diameter of the vessels, DtM = mean tangential diameter of the vessels, DtMx = maximum tangential diameter of the vessels, $\mathrm{Fpm}=$ minimum vessel frequency, $\mathrm{FpM}=$ mena vessel frequency, $\mathrm{FpMx}=$ maximum vessel frequency, $A v=$ vessel area and $F v=$ vessel fraction.

\section{Rugosidade superficial}

A Tabela 4 apresenta as médias de densidade aparente e dos parâmetros de rugosidade superficial das madeiras amazônicas em função do preparo da superfície e das classes de textura. A Tabela 5 apresenta as médias dos postos de Kruskal-Wallis dos parâmetros de rugosidade superficial em função das classes de textura das madeiras nas faces aplainada e lixada.

O parâmetro $R_{a}$ apresentou diferenças significativas entre as superfícies aplainadas e lixadas para todas as madeiras estudadas (Tabela 4). A superfície lixada apresentou menor $\mathrm{R}_{\mathrm{a}}$ que a superfície aplainada, independente da espécie. Isso ocorre porque o lixamento uniformiza a superfície e minimiza a influência da estrutura anatômica (MOURA; HERNÁNDEZ, 2005).

Na superfície aplainada, a madeira de cerejeira apresentou a maior $\mathrm{R}_{\mathrm{a}^{\prime}}$ entretanto não se diferenciaram estatisticamente das madeiras de abiurana, garapeira, cedro e cumaru. A madeira de jequitibá apresentou a menor $\mathrm{R}_{\mathrm{a}^{\prime}}$ entretanto, não se diferenciou das madeiras de angelim e angelim pedra. 





Tabela 5. Média dos postos de Kruskal-Wallis da rugosidade superficial em função da textura da madeira nas superfícies aplainada e lixada.

Table 5. Kruskal-Wallis rank means of the surface roughness as related to wood texture on planning and sanding surfaces.

\begin{tabular}{lccccc}
\hline \multicolumn{5}{c}{ Superfície aplainada } \\
\hline Textura & $\mathbf{R a}$ & $\mathbf{R z}$ & $\mathbf{R t}$ & $\mathbf{R p}$ & $\mathbf{R v}$ \\
\hline Fina & $217,99 \mathrm{~b}$ & 233,20 & 246,83 & $209,35 \mathrm{~b}$ & 252,26 \\
Média & $241,69 \mathrm{~b}$ & 230,21 & 245,48 & $237,87 \mathrm{~b}$ & 235,41 \\
Grossa & $255,21 \mathrm{a}$ & 243,34 & 226,68 & $264,39 \mathrm{a}$ & 223,85 \\
Valor H & $7,86^{*}$ & $0,74^{\text {ns }}$ & $2,48^{\text {ns }}$ & $17,07^{*}$ & $4,56^{\text {ns }}$ \\
\hline Superfície lixada & & & & & $211,68 \mathrm{~b}$ \\
\hline Fina & $166,56 \mathrm{~b}$ & $197,19 \mathrm{~b}$ & $201,30 \mathrm{~b}$ & $180,94 \mathrm{~b}$ & $351,49 \mathrm{a}$ \\
Média & $365,31 \mathrm{a}$ & $354,32 \mathrm{a}$ & $342,82 \mathrm{a}$ & $335,26 \mathrm{a}$ & $342,84 \mathrm{a}$ \\
Grossa & $374,35 \mathrm{a}$ & $353,34 \mathrm{a}$ & $352,89 \mathrm{a}$ & $370,36 \mathrm{a}$ & $85,44^{* *}$ \\
Valor H & $205,48^{* *}$ & $118,18^{* *}$ & $108,51^{* *}$ & $163,83^{* *}$ & \\
\hline
\end{tabular}

Médias com a mesma letra não são estatisticamente diferentes entre si. * $P>0,05$. ** $P>0,0 I$. ${ }^{\text {ns }}$ Não significativo. $R=$ rugosidade média, $R=$ rugosidade total, $R_{t}=$ rugosidade máxima, $R_{p}=$ distância vertical do pico mais alto no comprimento de medição e $R_{v}=$ vale mais profundo no comprimento de medição.

Na superfície lixada, a cerejeira apresentou a maior $\mathrm{R}_{\mathrm{a}^{\prime}}$ não se diferenciando estatisticamente das madeiras de angelim, cedro e cumaru. Entretanto, a cerejeira diferenciou-se da madeira de garapei$\mathrm{ra}$, a qual por sua vez, apresentou uma $\mathrm{R}_{\mathrm{a}}$ similar àquela das madeiras de jequitibá e abiurana.

Na Tabela 5, observa-se que a textura da madeira apresenta um efeito significativo na $R_{a}$ das duas condições de preparo. Na superfície aplainada, as madeiras de textura grossa apresentaram os maiores valores de $\mathrm{R}_{\mathrm{a}^{\prime}}$ enquanto que as madeiras de textura fina e média não se diferenciam estatisticamente entre si. Na superfície lixada, o efeito da textura na $\mathrm{R}_{\mathrm{a}}$ é mais perceptível em que as madeiras de textura fina (abiurana, garapeira e jequitibá) apresentaram os menores valores de $\mathrm{R}_{\mathrm{a}^{\prime}}$ diferenciando-se das madeiras de textura média e grossa. Esses resultados corroboram com aqueles observados por Thoma et al. (2015), em que as madeiras de textura fina apresentaram menor rugosidade superficial que as madeiras de textura grossa. Segundo Thoma et al. (2015), em superfícies lixadas a diferença entre os parâmetros de rugosidade superficial é explicada pela textura da madeira e sua uniformidade em relação ao tamanho e distribuição dos poros.

As madeiras estudadas apresentaram densidades aparentes médias variando entre $0,575 \mathrm{~g} . \mathrm{cm}^{-3}$ (cedro) e 0,974 g.cm ${ }^{-3}$ (angelim); entretanto nenhuma correlação foi encontrada entre a densidade e os parâmetros de rugosidade. Alguns autores afirmam que madeiras com maiores densidades produzem superfícies mais lisas após a usinagem e, consequentemente, menores valores de $\mathrm{R}_{\mathrm{a}}$. Teles et al. (2016) encontraram uma correlação negativa entre a densidade aparente e os parâmetros de rugosidade $\mathrm{R}_{\mathrm{a}}$ e $\mathrm{R}_{\mathrm{z}}$ para a madeira de cumaru em que a qualidade da superfície foi melhor para as peças de madeira de maior densidade.

Menores valores de rugosidade superficial são características desejáveis para madeiras destinadas à produção de móveis, por possibilitar uma superfície mais homogênea para aplicação de revestimentos. Entretanto, essa característica da superfície não deve ser vista isoladamente para que a madeira seja ou não apta para produção de móveis.

$\mathrm{O}$ parâmetro $\mathrm{R}_{\mathrm{z}}$ apresentou diferenças significativas entre as superfícies aplainadas e lixadas para todas as madeiras estudadas (Tabela 4). Na superfície aplainada, a madeira de abiurana apresentou o maior valor absoluto de $\mathrm{R}_{z^{\prime}}$ entretanto, ela diferenciou-se somente da madeira de jequitibá. As demais espécies apresentaram $\mathrm{R}_{\mathrm{z}}$ similar às madeiras de abiurana e jequitibá. A textura da madeira não afetou a $R_{z}$ da superfície aplainada (Tabela 5). A superfície lixada apresentou uma menor rugosidade média que a superfície aplainada em todas as madeiras.

A classe de textura da madeira afetou significativamente a $R_{z}$ das madeiras na superfície lixada (Tabela 5). As madeiras de textura fina (abiurana, garapeira e jequitibá) apresentaram os menores valores de $\mathrm{R}_{\mathrm{z}}$ diferenciando-se das madeiras de textura média e grossa.

$\mathrm{O}$ parâmetro $\mathrm{R}_{\mathrm{t}}$ apresentou diferenças significativas entre as superfícies aplainadas e lixadas para as madeiras estudadas, com exceção da madeira de angelim pedra (Tabela 4). Neste caso, na superfície lixada foram encontrados os menores valores de $\mathrm{R}_{t}$ quando comparada a superfície aplainada.

Na superfície aplainada, a madeira de angelim apresentou o maior valor absoluto de $\mathrm{R}_{\mathrm{t}^{\prime}}$ não diferenciando estatisticamente das madeiras de abiurana, garapeira, cedro e cumaru. A madeira de angelim pedra apresentou o menor valor absoluto de $\mathrm{R}_{\mathrm{t}^{\prime}}$ entretanto, não diferenciou-se das madeiras de jequitibá e cerejeira. A textura da madeira não afetou a $\mathrm{R}_{\mathrm{t}}$ da superfície aplainada (Tabela 5). 
Na superfície lixada, a textura afetou os valores de $\mathrm{R}_{\mathrm{t}}$ em que as madeiras de textura fina apresentam os menores valores (Tabela 5). As madeiras de textura média (cedro) e grossa (angelim, angelim pedra, cerejeira e cumaru) não se diferenciaram entre si. Na classe de textura fina, a madeira de jequitibá apresentou a menor $R_{t}$. O parâmetro $R_{t}$ é o maior valor das rugosidades parciais no percurso de medição sendo influenciado pelos parâmetros $R_{p} e R_{v}$.

$\mathrm{O}$ parâmetro $\mathrm{R}_{\mathrm{p}}$ apresentou diferenças significativas entre as superfícies aplainadas e lixadas para todas as madeiras estudadas (Tabela 4). Na superfície aplainada, a madeira de jequitibá apresentou o menor valor de $\mathrm{R}_{\mathrm{p}^{\prime}}$ diferenciando-se das demais madeiras. Na superfície lixada, observa-se o efeito da textura da madeira no parâmetro $\mathrm{R}_{\mathrm{p}^{\prime}}$ em que as madeiras de textura fina (abiurana, garapeira e jequitibá) apresentam os menores valores de $\mathrm{R}_{\mathrm{p}^{\prime}}$ diferenciando-se estatisticamente das demais madeiras.

$\mathrm{O}$ parâmetro $\mathrm{R}_{\mathrm{v}}$ apresentou diferenças significativas entre as superfícies aplainadas e lixadas para as madeiras estudadas, com exceção das madeiras de angelim e cumaru (Tabela 4). A superfície lixada apresentou menor $\mathrm{R}_{\mathrm{v}}$ quando comparada à superfície aplainada, com exceção das madeiras de angelim pedra e cumaru. Avaliando-se os perfis de rugosidade superficial típicos das madeiras de angelim pedra e cumaru nas duas condições de preparo da superfície, observa-se que o lixamento diminuiu os picos, mas não permite eliminar os vales presentes na superfície de ambas as espécies. Em madeiras de folhosas, o lixamento pode ocasionar o esmagamento das células, obstruindo os canais formados pelos vasos (MOURA; HERNÁNDEZ, 2005), o que contribui para diminuir a $\mathrm{R}_{\mathrm{v}}$. Entretanto, em madeiras mais densas tais como o cumaru e o angelim pedra (Tabela 4), há uma maior resistência mecânica dessas células não ocasionando essa obstrução. Segundo Gurau et al. (2005), em madeiras mais duras, a presença de vasos grandes e múltiplos interferem na rugosidade superficial devido à presença de vales profundos. De forma similar, observa-se no nosso estudo que as madeiras de angelim pedra e cumaru apresentaram diâmetros de vasos classificados como grandes e presença de vasos múltiplos, o que explicaria o fato do lixamento não interferir no parâmetro $R_{v}$ não ocasionando o entupimento desses elementos com o pó de lixa gerado no processo. Além disso, as análises de PCA agruparam as essas duas espécies em relação ao diâmetro dos poros (Figura 1).

Na superfície aplainada, a madeira de angelim pedra apresentou o menor valor absoluto de $\mathrm{R}_{\mathrm{v}^{\prime}}$ diferenciando-se somente das madeiras de jequitibá, cedro, cerejeira e cumaru. A textura não afetou a $R_{v}$ para a superfície aplainada. $O$ parâmetro $R_{v}$ foi influenciado mais pela frequência de vasos (poros $/ \mathrm{mm}^{2}$ ) do que pelo diâmetro tangencial do lume dos vasos, pois a madeira de angelim pedra apresentou o maior diâmetro tangencial médio e a menor média de frequência de poros.

A textura afetou o parâmetro $R_{v}$ na superfície lixada (Tabela 5). As madeiras de textura fina apresentaram os menores valores de $\mathrm{R}_{v^{\prime}}$ quando comparadas às madeiras de textura média e grossa. Entretanto, $\mathrm{o} \mathrm{R}_{\mathrm{v}}$ da madeira de garapeira foi estatisticamente diferente somente das madeiras de cumaru, com maior valor absoluto de $\mathrm{R}_{v^{\prime}}$ e jequitibá, com menor valor absoluto de $\mathrm{R}_{\mathrm{v}}$. De forma geral, os resultados mostram que a textura da madeira apresenta um efeito maior nas superfícies lixadas que na aplainada.

\section{CONCLUSÕES}

A análise da componente principal separou as madeiras em três grupos em que o diâmetro tangencial médio e a frequência média dos poros foram as variáveis mais importantes.

O lixamento diminuiu a rugosidade superficial das madeiras para todos os parâmetros, exceto para o parâmetro $R_{v}$ das madeiras de angelim pedra e cumaru, por apresentarem densidades altas e diâmetros tangenciais de vasos grandes.

A textura afetou os parâmetros $\mathrm{R}_{\mathrm{a}}$ e $\mathrm{R}_{\mathrm{p}}$ das superfícies aplainadas e todos os parâmetros de rugosidade na superfície lixada.

As madeiras de textura fina apresentaram menor rugosidade superficial que as madeiras de textura grossa.

A estrutura anatômica afetou a rugosidade superficial das madeiras nos parâmetros relacionados à profundidade, tendo como destaque a frequência média de poros. A separação dos grupos pela análise da componente principal, das variáveis anatômicas, correspondeu parcialmente ao agrupamento dado pelos valores médios de rugosidade superficial das madeiras amazônicas estudadas. 


\section{REFERÊNCIAS BIBLIOGRÁFICAS}

ASTM - AMERICAN SOCIETY FOR TESTING AND MATERIALS. D 2395-93: Standard test methods for specific gravity of wood and wood-based materials. ASTM Standards, Philadelphia, 1999. p. 350-357.

ABNT - ASSOCIAÇÃO BRASILEIRA DE NORMAS TÉCNICAS. NBR ISO 4287 Especificações geométricas dos produtos (GPS) - rugosidade: método do perfil - termos, definições e parâmetros da rugosidade. Rio de Janeiro, 2002. 5 p.

BAJIC, D.; LELA, B.; ZIVKOVIC, D. Modeling of machined surface roughness and optimization of cutting parameters in face milling. Metalurgija, v. 47, n. 4, p. 331-334, 2008.

BHUSHAN, B. Surface roughness analysis and measurement techniques. In: . Modern Tribology Handbook. Boca Raton: CRC Press LLC, 2001.

CORADIN, V. T. R.; MUÑIZ, G. I. B. Normas para procedimentos em estudos de anatomia de madeira: I. Angiospermae. II. Gimnospermae. Brasília: IBAMA/LPF, 1992. (Série Técnica, 15).

CSANÁDY, E.; MAGOSS, E.; TOLVAJ, L. Quality of machined wood surfaces. Basel: Springer International Publishing Switzerland, 2015.

MOURA, L. F.; HERNÁNDEZ, R. E. Evaluation of varnish coating performance for two surfacing methods on sugar maple wood. Wood and Fiber Science, v. 37, p. 355-366, 2005.

FOREST PRODUCTS LABORATORY. Wood handbook: wood as an engineering material. Madison: U.S. Department of Agriculture, 2010, 508 p. (General Technical Report FPL-GTR-190).

FORM Talysurf Series operator's handbook. London: RANK Taylor Hobson Limited, 1985. 359 p. (Publication $n^{\circ}$ RTH-HB-100)

GURAU, L.; WILLIAMS, H. M.; IRLE, M. Separation of processing roughness from anatomical irregularities and fuzziness to evaluate the effect of grit size on sanded European oak. Forest Product Journal, LaGrange, v. 57, n.1/2, p.110-115, 2007.

HERNÁNDEZ, R. E.; LLAVÉ, A. M.; KOUBAA, A. Effects of cutting parameters on cutting forces and surface quality of black spruce cants. European Journal of Wood and Wood Products, v. 72, p. 107-116, 2014.

KILIC, M.; HIZIROGLU, S.; BURDURLU, E. Effect of machining on surface roughness of wood. Building and Environment, v. 41, n. 8, p. 1074-1078, 2006.

MACHADO, A. R.; ABRÃO, A. M.; COELHO, R. T.; da SILVA, M. B.; RUFFINO, R. T. Teoria da usinagem dos materiais. São Paulo: Editora Blucher, 2009. 384 p.

MAGOSS, E. General regularities of wood surface roughness. Acta Silvatica et Lignaria Hungarica, v. 4, p. 81-93, 2008.

MARTINS, S. A.; FERRAZ, J. M; SANTOS, C. M. T; DEL MENEZZI, C. H. S.; SOUZA, M. R. Efeito da usinagem na rugosidade da superfície da madeira de Eucalyptus benthamii. Floresta e Ambiente, Seropédica, v. 18, n. 2 , p. 135-143, 2011.

PETROPOULOS, G. P. Multi-parameter analysis and modeling of engineering surface texture. Journal of Achievements in Materials and Manufacturing Engineering, v. 24, n. 1, p. 91-100, 2007. 
Pereira et al. - Rugosidade da superfície de madeiras amazônicas

TELES, R. F.; COSTA, A. F.; SOUZA, M. R. Análise da influência do tratamento preservativo na cor e rugosidade de superfícies de madeiras tropicais. Floresta e Ambiente, Seropédica, v. 23, n. 1, p. 100-108, 2016.

THOMA, H.; PERI, L.; LATO, E. Evaluation of wood surface roughness depending on species characteristics. Maderas. Ciencia y Tecnologia, Concepcion, v. 17, n. 2, p. 285-292, 2015.

UGULINO, B.; HERNÁNDEZ, R. E. Assessment of surface properties and solvent-borne coating performance of red oak wood produced by peripheral planning. European Journal of Wood and Wood Products, v. 75 , n. 4, p. 581-593, 2017.

ZHONG, Z. W.; HIZIROGLU, S.; CHAN, C. T. M. Measurement of the surface roughness of wood-based materials used in furniture manufacture. Measurement, v. 46, p. 1482-1487, 2013.

Recebido em 06/07/2017

Aceito em 12/12/2017 